\title{
Enseñanza de la(s) lengua(s) en Colombia desde una perspectiva glotopolítica*
}

\author{
MIREYA CISNEROS ESTUPIÑÁN** \\ MIGUEL ÁNGEL MAHECHA BERMÚDEZ***
}

Recepción: 5 de septiembre de 2019

Aprobación: 20 de diciembre de 2019

Forma de citar este artículo: Cisneros, M \& Mahecha, M. A. (2020). Enseñanza de la(s) lengua(s) en Colombia desde una perspectiva glotopolítica. Cuadernos de Lingüística Hispánica, (35), 157-178.

https://doi.org/ 10.19053/0121053X.n35.2020.10553

* Este artículo forma parte de los avances de un proyecto mayor titulado "Introducción de los estudios glotopolíticos en la formación profesional de docentes de lenguaje en Colombia”, inscrito, sin financiación, en la Vicerrectoría de Investigaciones de la Universidad Tecnológica de Pereira, con el código 4-19-3.

** Doctora en Ciencias de la Educación, Universidad del Cauca; Magister en Lingüística Española, Instituto Caro y Cuervo; Profesora del área de la Lingüística en la Licenciatura de Literatura y Lengua castellana y de la Maestría en Lingüística de la Universidad Tecnológica de Pereira. Correo electrónico: mireyace@gmail.com Bhttps://orcid.org/0000-00025519-7192

*** Magister en Lingüística Española, Instituto Caro y Cuervo; Profesor del área de Lingüística, programa de Literatura y Lengua Castellana, Universidad Surcolombiana, Neiva. Correo electrónico: miguelangel.mahecha@usco.edu.co @https://orcid. org/0000-0002-2263-2362 


\section{Resumen}

En este artículo se presentan reflexiones glotopolíticas en relación con el plurilingüismo y el bilingüismo en Colombia, así como las particularidades de una aproximación tanto sociolingüística como glotopolítica. También se plantea la necesidad de implementar en nuestro país una política y una planificación lingüísticas fundamentadas en criterios glotopolíticos que permitan el desarrollo equitativo de las lenguas que forman parte de la realidad social, cultural y cotidiana en Colombia.

Palabras clave: Colombia; glotopolítica; políticas lingüísticas; bilingüismo;multilingüismo; sociolingüística.

\section{Teaching of the Language in Colombia from a Glotopolitical Perspective}

\section{Abstract}

This paper presents glotopolitical reflections in relation to multilingualism and bilingualism in Colombia, as well as the characteristics of both sociolinguistic and glotopolitical approach. It also raises the need to implement in our country linguistic policy and planning based on glotopolitical criteria enabling equitable development of languages that are part of the social, cultural and everyday reality in Colombia.

Keywords: Colombia; glotopolitics; linguistic politics; bilingualism; multilingualism;sociolinguistics. 


\section{Enseigner les langues en Colombie dans une perspective glotopolitique}

\section{Résumé}

Cet article présente des réflexions glotopolitiques en rapport avec le multilinguisme et le bilinguisme, ainsi que les particularités d'une approche aussi bien sociolinguistique que glotopolitique, en Colombie. Il propose également, de mettre en œuvre une politique et une planification linguistiques, basées sur des critères glotopolitiques qui permettent le développement équitable des langues qui font partie de la réalité sociale, culturelle et quotidienne en Colombie.

Mots-clés: Colombie; glotopolitique; politiques linguistiques; bilinguisme; multilinguisme; sociolinguistique.

\section{Ensino de línguas na Colômbia de uma perspectiva glotopolítica}

\section{Resumo}

Este artigo apresenta reflexões glotopolíticas em relação ao multilinguismo e bilinguismo na Colômbia, bem como as particularidades de uma abordagem sociolinguística e glotopolítica. Também é necessário implementar em nosso país uma política e um planejamento linguísticos baseados em critérios glotopolíticos que permitam o desenvolvimento equitativo de idiomas que fazem parte da realidade social, cultural e cotidiana da Colômbia.

Palavras-chave: Colômbia; glotopolítica; políticas linguísticas; bilinguismo; multilinguismo; sociolinguística. 


\section{Introducción}

Al ser el español la lengua de comunicación de la mayor parte de la población colombiana, empleada en la cotidianidad y en los contextos de prestigio social, es esperable que en los espacios académicos de enseñanza de y en esta lengua, se implemente una dinámica glotopolítica que cumpla dos funciones claras: la primera, sensibilizar a la comunidad educativa de que en la realidad lingüística colombiana, el español es una de las tantas lenguas que se hablan en el territorio nacional, que aporta una manera de ver el mundo complementaria con las demás, consolidando la identidad colombiana ${ }^{1}$. La segunda, proponer métodos y recursos didácticos que fortalezcan el desarrollo de las competencias comunicativas (hablar, escuchar, leer y escribir) en la lengua materna.

Las circunstancias del mundo actual exigen una visión diferente frente a la escogencia y utilización de las lenguas en los diversos territorios. Es por esto que en el ámbito de los estudios contemporáneos sobre el lenguaje se justifica una perspectiva glotopolítica capaz de analizar políticas lingüísticas pertinentes y viables, así como orientar a distintos colectiva social sobre medidas de planeamiento del lenguaje (Arnoux, 2008, p. 1; Calvet, 1993, p.111; Chaves-0'Flynn, 2007, p. 22). Su importancia radica en revelar, proponer y orientar decisiones políticas en torno a la producción y reproducción de representaciones sociales, ideologías y regulaciones sobre la lengua a nivel local, regional e internacional (Calvet, 1987; Arnoux, 2016).

Las ideologías posicionan al español en el mapa mundial de las lenguas y le otorgan capacidad de agencia y transformación de las estructuras sociales; al mismo tiempo, las acciones políticas sobre el lenguaje llegan a jerarquizar las lenguas en función de intereses económicos, incidir en fenómenos de exclusión, discriminación y explotación, y apoyar o despreciar proyectos regionales de unión y colaboración entre países (Cisneros y Olave, 2018; Instituto Cervantes, 2010; Lastra, 2003; Vásquez, 2008).

1 Artículo 7. El Estado reconoce y protege la diversidad étnica y cultural de la Nación colombiana. Artículo 10. El castellano es el idioma oficial de Colombia. Las lenguas y dialectos de los grupos étnicos son también oficiales en sus territorios. La enseñanza que se imparta en las comunidades con tradiciones lingüísticas propias será bilingüe. Artículo 13. Todas las personas nacen libres e iguales ante la ley, recibirán la misma protección y trato de las autoridades y gozarán de los mismos derechos, libertades y oportunidades sin ninguna discriminación por razones de sexo, raza, origen nacional o familiar, lengua, religión, opinión política o filosófica. 
Mientras en Europa hay una larga tradición en estudios glotopolíticos ${ }^{1}$ y en Latinoamérica un creciente desarrollo (Del Valle, 2017), en Colombia no ha tenido la suficiente atención y dedicación por parte de los investigadores. Puede afirmarse que la tradición lingüística en el país tiene grandes fortalezas en el estudio descriptivo de la lengua española (Instituto Caro y Cuervo, 2018) y en los estudios dialectológicos (Lozano, 2012; Montes, 1995, 2000) y etnolingüísticos (González de Pérez, 2011; Patiño, 2000; Trillos, 1998, 2001, Trillos y Etxebarria, 2002; Rodríguez, 1996), y un recorrido menor -aunque no despreciable- en los estudios discursivos (Pardo, 2011; Soler, 2014). Esos énfasis en la investigación lingüística no alcanzan, de manera suficiente, la reflexión sobre ideologías lingüísticas con perspectivas glotopolíticas² (Cisneros y Olave, 2018).

Particularmente en el ámbito de la formación de docentes de lengua materna, la perspectiva glotopolítica no figura en los currículos actuales, como lo comprueba una investigación reciente del grupo Estudios del Lenguaje y la Educación (Cisneros, Olave y Rojas, 2016) así como Mahecha (en prensa). El efecto negativo de esta carencia es la dificultad para desarrollar el campo de estos estudios en la academia colombiana y el retraso de los aportes que puede realizar el país a esa línea de trabajo de la lingüística contemporánea y al diseño de políticas lingüísticas adecuadas y coherentes con la realidad social y cultural del país en materia de plurilingüismo y bilingüismo.

\section{Glotopolítica y plurilingüismo en Colombia}

Nos parece que la definición de "glotopolítica" ofrecida por Baggioni (1986) es más clara que la propuesta en diferentes trabajos por Arnoux y su equipo, y encaja con más facilidad en nuestra realidad lingüística nacional:

Si definimos la glotopolítica como la reflexión explícita del lingüista sobre una racionalización de los intercambios lingüísticos entre 0 al interior de las comunidades lingüísticas (cuyos contornos y realidad sontambién objeto de reflexión por parte del lingüista), convendremos en que esta reflexión se presenta bajo un doble aspecto, el de una producción de conocimiento y el de formulación de propuestas de intervención en el campo político. Desde esta perspectiva no tiene sentido establecer límites definidos entre ciencia e ideología, producción

1 Consúltense los siguientes documentos: (i) La política editorial y los diferentes números temáticos de la revista Glottopol de la Universidad de Rouen (Francia) http://glottopol.univ-rouen.fr/ (ii) Baggioni, D. (1986) Préhistoire de la glottopolitique dans la linguistique européenne, de J. G. Herder au Cercle linguistique de Prague; (iii) Guespin y Marcellesi (2019) Hacia la glotopolítica; (iv) Certeau y Revel (1975) Une politique de la langue. La Révolution française et les patois.

2 Los trabajos (referenciados aquî) de Rico (1981, 1994, 1998), Mahecha (2010. 2015, 2017), Pineda (1997, 2000) y Villa (2002), han insistido en este punto y han hecho sugerencias para el desarrollo glotopolítico. 
de conocimiento e ideologías mistificadoras / justificadoras, que, en mi opinión, podrían tener la ventaja de ubicar la reflexión del lingüista en su contexto histórico, político y social. (p. 35, traducción nuestra).

Si bien es cierto que en Colombia la perspectiva glotopolítica no ha tenido el mismo impulso que en otros países latinoamericanos, no se puede negar que en diferentes centros universitarios se hayan postulado iniciativas individuales, aisladas, que han tratado de describir la realidad lingüística nacional echando mano de algunos elementos glotopolíticos ( $c f$. nota 3). Para ello, se han tomado como objetos de estudio el bilingüismo, el multilingüismo y la enseñanza de lenguas y se han descrito con categorías de análisis glotopolítico (Arismendi, 2016; Buitrago, García y García, 2011; ChávesOFlynn, 2017; Figueroa, 2007; Mahecha, 2015).

En 1992, la Presidencia de la República le solicitó a la Universidad de los Andes la traducción de la Constitución Política de Colombia a las lenguas indígenas. El profesor de esa universidad, Jon Landaburu, se encargó de dirigir una traducción colectiva en las comunidades indígenas con talleres en siete lenguas (Landaburu, 1997; Oróstegui, 2008) ${ }^{4}$. Allí, siete lingüistas indígenas nativos hablantes de su lengua, y formados por él y por investigadores del Instituto Caro y Cuervo, con la asistencia de siete abogados indigenistas, trabajaron durante dos años para lograr la traducción de una terminología jurídica para personas que viven en zonas rurales, que son pescadores o campesinos.

Este intento por cumplir con los requerimientos de la Constitución de 1991, según la cual el castellano es el idioma oficial de Colombia, pero las lenguas y dialectos de los grupos étnicos son también oficiales en sus territorios, planteó una serie de problemas filosóficos, semánticos y jurídicos al tratar de articular un pensamiento urbano letrado y judeocristiano (la secuencia clásica del Mediterráneo como la llamaba Fals Borda), con mundos muy diferentes. Así, más que la traducción de la Constitución como tal, fue la reflexión colectiva de los indígenas sobre sus propias leyes y cómo se articulan estas con las de los otros territorios y formas de vida. Esto permitió, en ese momento, y por los pocos que conocieron tal situación, una toma de conciencia moral, política y cultural (Cisneros, 2016). Sin embargo, este intento glotopolítico se ha quedado en suspenso en las tres últimas décadas.

De acuerdo con datos del Atlas sociolingüístico de los pueblos indígenas. (UNICEF, 2009), Brasil es el que tiene más diversidad de pueblos indígenas con 241, que representan una población de 734.127 personas. Colombia, con 83

4 El wayuu o guajiro (Guajira), el nasa o páez (Cauca), guambiano (Cauca), arhuaco o ika (Sierra Nevada de Santa Marta), ingano (Putumayo), el kamsá (Sibundoy, Putumayo), el cubeo del Amazonas (Vaupés). 
(1.392.623 habitantes) es el segundo país con más cantidad de pueblos, seguido por México con 67 (9.504.184 personas) y por Perú, que tiene 43 pueblos distintos que representan 3.919.314 habitantes.

En el otro extremo, se encuentra El Salvador, que tiene 3 pueblos indígenas (13.310 personas), Belice con 4 (38.562 habitantes) y Surinam con 5 (6.601 personas). En el caso del Caribe insular, como Antigua y Barbuda, Trinidad y Tobago, Dominica y Santa Lucía, hay pocos datos sobre la supervivencia de pueblos nativos, pero existen reivindicaciones de identidad indígena en el ámbito local.

Por otra parte, Bolivia, Guatemala y Belice destacan por ser los países donde los indígenas representan porcentajes más altos sobre la población total, con el $66,2 \%$, el 39,9\% y el 16,6\% respectivamente. En cambio, países como El Salvador, Brasil, Argentina, Costa Rica, Paraguay y Venezuela registran un bajo porcentaje de población indígena (entre $0,2 \%$ y 2,3\%). No obstante, en la mayoría de países latinoamericanos la población indígena va del 3\% al 10\% del total de ciudadanos.

México, Bolivia, Guatemala, Perú y Colombia reúnen al 87\% de indígenas de América Latina y el Caribe, con una población que se sitúa entre un máximo de 9.500.000 (México) y un mínimo de 1.300 .000 habitantes (Colombia). El restante 13\% de población indígena reside en 20 Estados distintos. (Negrillas nuestras)

A pesar de que la realidad lingüística colombiana posee una destacada variedad idiomática en la región, ya que además del castellano existen 68 lenguas habladas por grupos étnicos: 65 lenguas indígenas, dos criollas (una de base española, el "palenquero", y la otra de base inglesa, el "criollo sanandresano"), la lengua de los gitanos (el romaní) y la lengua de la comunidad de sordos, esta peculiaridad natural no se ha considerado seriamente por parte de los estudiosos colombianos para llamar la atención en torno a la urgencia de crear y consolidar una conciencia glotopolítica.

Desde una perspectiva lingüístico-tipológica, las lenguas indígenas colombianas tienen estructuras tan complejas como la de cualquier idioma del mundo, a pesar de ser ágrafas. La mayoría está dispersa por los lugares más inhóspitos y periféricos, precisamente los menos intervenidos durante los periodos de la conquista y la colonia y en la actualidad, los más desplazados y con graves problemas de orden público e invasión de sus territorios. Algunas lenguas son tonales (así como el chino), algunas son flexionales (como lo es el griego antiguo), otras son de tipo aglutinante (como lo es el húngaro). Hay trece familias lingüísticas, más ocho lenguas aisladas que hasta el momento ha resultado difícil relacionar con otras. Por lo tanto, hay un total de 21 raíces lingüísticas diferentes (Cisneros, 2016). 
La ausencia de políticas lingüísticas en nuestro país a lo largo de su historia ha traído como consecuencia la desaparición progresiva de lenguas como la muisca que, aunque llegó a ser lengua general o lengua franca, o sea materia obligada para los religiosos que aspiraban a adoctrinar en suelo americano - enseñada incluso en el Colegio Mayor de Nuestra Señora del Rosario, en Bogotá-, ha desaparecido desde el siglo XVIII. Así, vemos con dolor y con impotencia como más de la mitad de las lenguas oficiales de Colombia, según su Constitución Política, se están perdiendo.

\section{Bilingüismo en ausencia de una fundamentación glotopolítica}

La pregunta por el estado del bilingüismo en Colombia se inscribe inicialmente en las propuestas teóricas que se centran en la relación entre el español como lengua oficial dominante y las lenguas indígenas y criollas como lenguas igualmente oficiales pero sometidas políticamente (diglosia). Es en este dominio en donde se encuentra el mayor número de miradas políticas desde el campo gubernamental e investigativas desde el campo de la lingüística, a pesar de que la realidad social colombiana se fundamenta en la coexistencia natural de hablantes de aproximadamente 68 lenguas, como lo referíamos en el apartado anterior.

Los trabajos investigativos sobre bilingüismo proceden de la didáctica de la lengua extranjera en donde los postulados sociolingüísticos no son tenidos en cuenta con las fundamentaciones teóricas y prácticas que lo ameritan. De ese modo, se desconoce que la lengua extranjera no es o no se hace funcional en nuestro territorio y su enseñanza, en mucho, se siente como imposición en los contextos educativos y, por ende, no consigue el interés por parte de los estudiantes para ser aprendida. No se desconoce que hay trabajos interesantes que se ocupan de la relación español/lengua extranjera (con mayor atención en el inglés) en cuanto a procesos de calco semántico, de la traducción e incluso la interferencia.

En el afán de constituir un bilingüismo desde políticas gubernamentales, con atención a las lenguas extranjeras, no se ha ilustrado suficientemente la realidad sociolingüística colombiana en donde los hablantes nativos de las lenguas indígenas, las lenguas criollas, la lengua de los limitados auditivos y el romaní han debido -por razones glotopolíticas- apoyarse en el español no solo como lengua vehicular, sino también, en caso extremo, como lengua de cultura. De esta forma, estos hablantes no han constituido un espacio lo suficientemente marcado para "nacionalizar" su visión del mundo.

De ahí que el fenómeno del bilingüismo ha sido abordado en nuestro medio tan solo desde un enfoque glotopolítico interpretado, como lo acabamos de mencionar, de 
una manera que conviene a intereses particulares ${ }^{5}$, obviando los referentes naturales inmediatos de la comunicación cultural colombiana, que involucran aspectos socio y psicolingüísticos. Más bien se ve, de alguna forma, una tendencia a la glotofagia.

De igual forma, el querer implantar a manera de gestión in vitro políticas culturales carentes de una sólida fundamentación científica, que privilegian, entre otras cosas, un idioma extranjero (el inglés), en detrimento de otras lenguas (autóctonas [lenguas indígenas] y extranjeras) es lo que ha impedido consolidar una política y una planificación lingüísticas acordes con la realidad social colombiana, que ameritan procesos glotopolíticos adecuados.

La evaluación de las políticas lingüísticas -con sus correspondientes planificaciones - la economía de lenguas y la glotopolítica, entre otras tantas, constituyen el marco de referencia más adecuado para entender cómo la política lingüística nacional y sus respectivas consecuencias en materia de enseñanza de las lenguas pueden ser consideradas desde el punto de vista de asignación de presupuestos y de distribución de recursos; en otra palabras, es hablar en términos de eficiencia, competitividad y prospectiva. Esta relación afectaría directamente la configuración, por ejemplo, de los proyectos pedagógicos de las facultades, el papel de la lengua extranjera que se impone, como es el caso del inglés no solo frente a otras lenguas, sino frente al español, pues la argumentación que justifica su enseñanza/aprendizaje radica más en el hecho de los diferenciales salariales que provienen del manejo de esta lengua y el prestigio social de quien la maneja, que de la consideración de aspectos lingüísticos que caracterizan la realidad social regional (cfr. los trabajos de Pardo (2001), Rico (1998) y Mahecha (2015).

Las políticas lingüísticas se consideran, generalmente, como orientaciones 0 instrumentos estatales y casi siempre desde una perspectiva de organización de la defensa de una 0 varias lenguas nacionales/regionales (política lingüística interna) o de expansión de dicha 0 dichas lenguas (política lingüística externa). Remiten entonces a los esfuerzos públicos que pretenden modificar el estatus relativo y el uso de una o varias lenguas. En el contexto de las construcciones nacionales, la unificación lingüística como reto administrativo y simbólico se ha plasmado a veces en el monolingüismo y otras veces en el reparto de territorios; y la política lingüística explícita se interesa entonces, esencialmente, por la definición de las normas gramaticales oficiales de la lengua vehicular y por las condiciones de enseñanza de las lenguas minoritarias, dejando a lo implícito ocupar el espacio de un dejar hacer.

5 Del orden de lo político y lo económico. 
La irrupción del bilingüismo y plurilingüismo como dato consubstancial de las sociedades humanas perturba esta representación de la acción sobre las lenguas. Los actores sociales usuarios y difusores prescriptores, son numerosos y variados, van del individuo a los grupos organizados (administraciones, empresas, asociaciones), y el objeto se considera necesariamente plural en las relaciones instituidas entre las lenguas por el conjunto de las actividades sociales entre lo local y lo global (Hagège, 2012).

La cuestión que se plantea cuando se habla de bilingüismo y plurilingüismo y de sus componentes comunicativos y didácticos, así como de la intercomprensión o la educación a las lenguas, consiste en determinar si el desafío reside o no en los fundamentos, el diseño y la ejecución de políticas interlingüísticas explícitas soportadas principalmente en seis puntos (Mahecha, 2015):

- Grandes orientaciones teóricas y prácticas en materia de política lingüística

- Epistemología del plurilingüismo como objeto de política lingüística

- Espacio del plurilingüismo en las políticas lingüísticas internas y externas

- Situaciones lingüísticas geopolíticas y políticas de plurilingüismo

- Relación entre políticas lingüísticas y didácticas

- La intercomprensión en las políticas lingüísticas

Ahora bien, no se puede dejar de lado que es en el ámbito educativo, y más específicamente en el terreno de enseñanza de lenguas, donde mejor se percibe la dinámica de las políticas lingüísticas y sus correspondientes planificaciones, como lo propone Calvet $(1987,1993)$. Auroux y Bein (2015) lo manifiestan en un libro dedicado a este tema:

Las políticas lingüísticas han encontrado tradicionalmente en el sistema educativo un espacio importante de implementación. Las decisiones respecto de la enseñanza de lenguas maternas, segundas o extranjeras dependen de posiciones glotopolíticas explícitas o posibles de ser inferidas no solo de las prácticas áulicas, sino también, entre otros, de textos didácticos, evaluaciones, instrumentos lingüísticos que se destinan al uso escolar, antologías, resoluciones ministeriales, producciones de los alumnos o revistas de orientación docente. Las opciones que se privilegian en diferentes momentos y épocas e, incluso, en distintas instituciones corresponden tanto a las lenguas como a las variedades, los registros o las discursividades, y ponen en juego estrategias pedagógicas que 
parten de las representaciones dominantes acerca de los objetos lingüísticos, el sujeto escolarizado, los requerimientos sociales y los avances en las ciencias del lenguaje. (p. 10)

En nuestro caso colombiano no se ha diseñado una política lingüística acorde con la dinámica de la realidad sociocultural del país. Las iniciativas gubernamentales han tratado de esbozar políticas lingüísticas parciales en el ámbito educativo bajo el nombre de bilingüismo (Ley General de Educación, 1994), Programa Nacional de Bilingüismo, Programa de Fortalecimiento al Desarrollo de Competencias en Lenguas Extranjeras (PFDCLE), Programa Nacional de inglés Colombia very well, territorios bilingües, focalizando, no el fenómeno bilingüe como proceso cultural, sino la creación de condiciones ideales para que los colombianos demuestren niveles altos en inglés con el fin de ser competitivos en el mundo global.

De ese modo, lainterpretación y aplicación de los documentos oficiales colombianos, tales como la Constitución Política de 199116, la Ley 115 o Ley General de Educación, el Marco Común Europeo de Lenguas, los Estándares Básicos de Competencias en Lenguas Extranjeras, la política de protección de las lenguas de los grupos étnicos presentes en el territorio colombiano, la Ley 1381 del 25 de enero de 2010, conocida también como la Ley de Lenguas, así como de estudios científicos del área de la lingüística, entre otros, no han sido coherentes ni dialógicos con la realidad social colombiana.

Para lograr o acercarnos a los retos que implica la educación bilingüe, se hace necesario, entonces, ampliar los propósitos desde miradas sociolingüísticas y glotopolíticas, como lo veremos en el siguiente apartado.

\section{Sociolingüística y glotopolítica}

Si bien es de reconocer que la sociolingüística avanzó notablemente en la constitución de sus métodos interdisciplinarios para estudiar la relación entre lenguaje y sociedad, no alcanzó a aclarar su objeto de estudio y, más bien, se observan varios objetos y puntos de vista diferentes. De ese modo, podría considerarse la glotopolítica (entendida en el sentido de fundamento para la formulación de políticas lingüísticas) como uno de los objetos de la sociolingüística que se ha venido gestando y desarrollando, incluso sin mencionar esta denominación.

6 La Constitución Política de 1991 declara a Colombia como un país pluriétnico, plurilingüe y multicultural. En su artículo 10 dice: "[...] Las lenguas y dialectos de los grupos étnicos son también oficiales en sus territorios [...]". 
Así, por ejemplo, en Areiza, Cisneros y Tabares (2006) se encuentran capítulos como "Lenguas en contacto o lenguas en conflicto", "Extinción o muerte de una lengua", donde hay una ilustración glotopolítica enfática, pero no se menciona esta área de estudio e investigación que toma fuerza en otros países latinoamericanos, cabe mencionar los trabajos de Arnoux (2000, 2008, 2016), Del Valle (2017) y de IPOL.

Desde los estudios clásicos de sociolingüística en América, a manera de ejemplo se puede mencionar a Coseriu (1981), que indica la existencia de tres tipos de estudios sociolingüísticos:

a) estudio de la variedad de las lenguas, en la medida en que ésta no se consideraba ya en la dialectología (y, en parte también con respecto a lo ya considerado en la dialectología), b) estudio del "status" de diferentes tradiciones lingüísticas en una misma comunidad; c) estudio del grado de conocimiento y empleo de la lengua común por parte de los diferentes estratos socioculturales de una comunidad. (1981, p. 8)

Los propósitos de estos estudios podrían ir más allá de limitaciones teóricas del estudio del sistema formal de la lengua, si se entrecruzan y se abonan con políticas lingüísticas que los fortalezcan y los reconozcan.

En el ámbito europeo cabe mencionarse a Calvet, quien, en su obra $\mathrm{La}$ sociolinguistique (1993), reafirma la heterogeneidad de la sociolingüística en la historia de las ideas lingüísticas. Así, el primer capítulo del libro considera a Saussure como el defensor del paradigma de la lengua como sistema autónomo y cerrado, y a Meillet como defensor del paradigma de la lengua como el hecho social. Al final del libro, Calvet propone una alternativa para no caer en las trampas lexicales de la denominación y por ende de la definición, dejando en claro que podemos considerar la sociolingüística como un enfoque particular que intenta explicar la convergencia de variables tanto lingüísticas (entiéndase "sistema") como socioculturales de una comunidad dada, en un acto comunicativo específico; de ahí su valiosa síntesis: "el objeto de estudio de la lingüística no es solo la lengua o las lenguas sino la comunidad social en su aspecto lingüístico" (Calvet, 1993, 109) (traducción nuestra).

Esta proposición no ha sido discutida, al menos en las diferentes publicaciones que versan sobre este tema. Pero lo que sí queda suficientemente claro es que podemos considerar la sociolingüística como un enfoque particular que intenta explicar la convergencia de variables tanto lingüísticas (entiéndase "sistema") como socioculturales de una comunidad dada, en un acto comunicativo específico. Es por eso que el tema 
del bilingüismo como realidad social de una comunidad dada es uno de los tantos objetos de estudio de la disciplina mencionada. Y si a eso le añadimos que dentro de la sociolingüística hay una mirada más detallada que se ocupa del fenómeno en cuestión, esto es, del bilingüismo, entonces la perspectiva glotopolítica resultaría la más adecuada para hacerlo. La glotopolítica es una versión ampliada del enfoque politológico: políticas lingüísticas territoriales.

Por lo anterior, es preciso considerar que un fenómeno como el bilingüismo, 0 el multilingüismo, o los fenómenos variacionistas de una misma lengua son realidades sociales de comunidades de habla que se estudian desde la sociolingüística. Y si a esto le incluimos la relación con las políticas lingüísticas que atrofian o fortalecen usos de las lenguas y las variaciones, estamos frente a estudios glotopolíticos, tal y como lo asevera Baggioni (1986).

Como ya se mencionó y como lo reconoce el Ministerio de Cultura de Colombia (2013), la realidad lingüística colombiana es en esencia multilingüe y por ende multicultural, tal. Si bien es cierto que el multilingüismo y bilingüismo hacen parte de la realidad social colombiana, es conveniente decir también que dicho fenómeno no tiene un peso fuerte en la cotidianidad glotopolítica del país. Es en español que se vehicula la dinámica cultural nacional; las lenguas indígenas y criollas no han dejado de ser elementos exóticos que son mirados con indiferencia e incluso, ignorancia. Estos elementos deben ser tenidos en cuenta para la formulación de una política lingüística acorde con la realidad social colombiana.

En nuestro referente colombiano, abundan los comentarios superficiales sobre el fenómeno del bilingüismo. Dichos comentarios circulan libremente, convirtiéndose en una versión oficial de la realidad colombiana; son el fruto de una moda, de una información mal interpretada tanto por los medios de comunicación como por las entidades del Estado (p. ej., el Ministerio de Educación Nacional), y de una ausencia de análisis científico no solo por parte de los sociolingüistas, quienes llevan a cabo su labor generalmente en las universidades, sino también por parte de los entes gubernamentales, cuyo interés político desdibuja la armonía de la realidad social. Lo que sigue sería un borrador para proponer un anteproyecto de investigación que apunta al diseño de una política lingüística equitativa en nuestro medio regional y nacional, con el fin de que los agentes decisorios lleven a cabo una planificación acorde con nuestra realidad social. Queremos dejar en claro que no estamos en contra del inglés como lengua extranjera, siempre y cuando la selección de esta lengua obedezca a un estudio previo de las necesidades sociolingüísticas en lengua materna (ya sea el español u otra lengua hablada 
en el territorio nacional), para poder equilibrar el valor de ser "bilingüe" (hay que contar además con las actitudes emotivas de los hablantes).

El fenómeno del bilingüismo ha sido abordado en nuestro medio tan solo desde un enfoque glotopolítico interpretado, como lo acabamos de mencionar, de una manera que conviene a intereses particulares, obviando los referentes naturales inmediatos de la comunicación cultural colombiana. Además de esto, es visto desde una óptica restringida de la mecánica sociolingüística que deja de lado, entre otras cosas, el enfoque psicolingüístico. Es extraño que en el intento de hacer bilingües las regiones, cuestiones tales como los modelos teóricos de la lingüística cognitiva, la psicología del lenguaje, la ecología de las lenguas, por solo mencionar algunas, estén ausentes en los proyectos que se socializan desde los entes decisorios. Preguntas tales como iqué ventajas y desventajas cognitivas se encuentran en el fenómeno del bilingüismo? ¿Hay diferencias sociocognitivas entre individuos monolingües y bilingües (incluso plurilingües)? ¿Qué tipo de incidencia tienen variables sociolingüísticas como la edad, el sexo, el nivel educativo, la procedencia regional, el entorno inmediato y mediato en el aprendizaje de una nueva lengua? ¿Qué tipos de métodos son los más aconsejables cuando se trata de aprender una lengua en un medio exolingüe? No se aclara qué tipo de bilingüismo se practica en cada región: compuesto y coordinado, simultáneo y secuenciado, temprano y tardío, aditivo y sustrayente, etc. Más aún, ¿qué papel juegan en el proceso de aprendizaje los estilos y ritmos cognitivos de los estudiantes?

Ahora bien, se ha asumido que el bilingüismo es la capacidad de hablar en otra lengua diferente de la materna con el propósito de ser "más competentes y comunicativos" en un mundo globalizado. Pero si bien es cierto que el Ministerio de Educación Nacional de Colombia justifica la política de bilingüismo en el país alegando:

1) la necesidad de formar individuos competentes en un mundo globalizado:

El Gobierno Nacional tiene el compromiso fundamental de crear las condiciones para que los colombianos desarrollen competencias comunicativas en otra lengua [y que, en nuestro criterio, se deberían tener en cuenta lenguas de prestigio que han estado próximas a la realidad sociocultural colombiana como el francés y el portugués CyM]. Tener un buen nivel de inglés facilita el acceso a oportunidades laborales y educativas que ayudan a mejorar la calidad de vida. Ser competente en otra lengua es esencial en el mundo globalizado, el cual exige poderse comunicar mejor, abrir fronteras, comprender otros contextos, apropiar saberes y hacerlos circular, entender y hacerse entender, enriquecerse y jugar un papel decisivo 
en el desarrollo del país. Ser bilingüe amplía las oportunidades para ser más competentes y competitivos. (MEN, 2004) artificial:

y 2) la inclusión de elementos conceptuales que soportan una política lingüística

El bilingüismo se refiere a los diferentes grados de dominio con los que un individuo logra comunicarse en más de una lengua y una cultura. Estos diversos grados dependen del contexto en el cual se desenvuelve cada persona. Así pues, según el uso que se haga de otras lenguas distintas a la materna, éstas adquieren el carácter de segunda lengua o de lengua extranjera [...] La lengua extranjera, en cambio, es aquella que no se habla en el ambiente inmediato y local, pues las condiciones sociales cotidianas no requieren su uso permanente para la comunicación. Una lengua extranjera se puede aprender principalmente en el aula y, por lo general, el estudiante está expuesto al idioma durante períodos controlados. A pesar de no ser usada en circunstancias diferentes a las académicas, los estudiantes de una lengua extranjera pueden alcanzar altos niveles de desempeño para ser comunicadores eficientes cuando así lo requieran. (MEN, 2004. Las negrillas son nuestras),

Su objetivo es dar una idea integral del fenómeno bilingüe, pero falla cuando en el ejercicio de aplicación cotidiano se hace lo contrario al ideal esperado. Esta actitud contraria obedece tal vez a que las variables psicosociolingüísticas han sido dejadas de lado en la constitución de una política lingüística "bilingüe" para darle un énfasis exagerado al plano político económico (el de la competitividad):

En el contexto colombiano y para los alcances de esta propuesta, el inglés tiene carácter de lengua extranjera. Dada su importancia como lengua universal, el Ministerio de Educación ha establecido dentro de su política mejorar la calidad de la enseñanza del inglés, permitiendo mejores niveles de desempeño en este idioma. Así pues, se pretende que los estudiantes al egresar del sistema escolar, logren un nivel de competencia en inglés B1 (Pre intermedio) (MEN, 2004)

Con ello pretenden brindar un lenguaje común que permita a niños, niñas y jóvenes mayor acceso al mundo de hoy. Este hecho se ve confirmado por los datos suministrados por el Icfes respecto a las pruebas del 2004, según los cuales el noventa y nueve por ciento de los estudiantes seleccionaron el inglés en el examen de estado. (MEN, 2004) 
Y se establecen para ello medidas de competencia (lingüístico/comunicativa) en lengua inglesa, siguiendo, como es de suponerse, estándares internacionales como el marco de referencia europea para la enseñanza de lenguas.

En este contexto, "lengua extranjera" solo quiere decir "inglés", nada más. El programa Colombia Very Well, cuyos antecedentes se encuentran en el programa nacional de bilingüismo (2006-2010) y el programa de fortalecimiento al desarrollo de competencias en lenguas extranjeras (PFDCLE, 2010-2014), es un nuevo intento de formular una política lingüística que imponga y consolide la lengua inglesa en el referente cultural colombiano (Alonso, Martín y Gallo, 2015; Díaz et al, 2008). Es por eso que buscamos precisar las características de este fenómeno bilingüe desde la óptica sociolingüística (i.e. glotopolítica), intentando mostrar que se requiere de la consideración de diversas variables naturales de nuestra realidad social para poder formular una política lingüística eficaz.

Algunas de las ideas insinuadas han sido tratadas en los cursos de Sociolingüística para los estudiantes de programas de Lengua Extranjera, en los cursos de Lingüística para los estudiantes de programas de Lengua Castellana, Español y Literatura, Lenguaje y Cultura, entre otros, en los cuales se han utilizado manuales de sociolingüística, tales como Areiza, Cisneros y Tabares $(2006,2012,2018)$.

En Latinoamérica ya hay propuestas de trabajo lideradas principalmente desde Argentina y desde asociaciones como la ALFAL (Asociación de Lingüística y Filología de América Latina) y del IPOL (Instituto de Investigação e Desenvolvimento em Política Lingüística, Brasil). Cada país tiene la tarea de proponer investigaciones que apoyen, consoliden y proyecten las políticas del lenguaje en América Latina, y por eso en la clausura del Congreso de la Asociación de Lingüística y Filología de América Latina (ALFAL, 2017) se redactó, discutió y aprobó una convocatoria titulada "Por una ciencia y una educación superior pública, gratuita, crítica, humanista e intercultural, basada en modelos plurilingües de investigación y docencia". Los dos últimos párrafos se constituyen en el faro glotopolítico:

Abogamos asimismo por la preservación y el reforzamiento de modelos plurilingües de investigación, docencia y comunicación científica, basados en nuestras principales lenguas de integración latinoamericana, el español y el portugués, sin cerrarles nunca las puertas a las lenguas indígenas y de inmigración; y la apropiación vigorosa del inglés y de otras lenguas extranjeras a partir de las necesidades y en las modalidades definidas por nuestras comunidades científicas, impulsando la internacionalización de la investigación y enseñanza. 
Esto posibilitará fortalecer una relación con el inglés a partir de una posición no marcada por la subalternidad. Hacemos un llamado a las instancias políticas, a las instituciones gubernamentales de administración y fomento de la ciencia y educación superior y a las direcciones de universidades y otros centros de investigación a impulsar políticas acordes con los principios y orientaciones aquí formulados. (ALFAL, 2017)

\section{A manera de conclusión}

En nuestro referente colombiano, el discurso oficial hegemónico correspondiente al fenómeno del bilingüismo/multilingüismo carece de fundamentación sociolingüística, discursiva y glotopolítica, y lamentablemente es acogido por amplios sectores de la población, por las comunidades académicas, por las entidades gubernamentales y por los medios de comunicación, motivados por modas o informaciones mal interpretadas y sin análisis científicos, no solo por parte de los sociolingüistas, quienes llevan a cabo su labor generalmente en las universidades, sino también por parte de los entes gubernamentales, cuyo interés político desdibuja la armonía de la realidad social.

Los estudios glotopolíticos en Colombia están en mora de ser parte de la formación universitaria de los interesados y por ende involucrados en las actividades de la política nacional de bilingüismo, adelantada desde el Ministerio de Educación Nacional, las gobernaciones, las alcaldías y, por supuesto, las universidades, para unir fuerzas en la formulación de políticas lingüísticas acordes con la realidad sociolingüística de Colombia.

Resulta urgente, entonces, implementar desde las perspectivas sociolingüística y glotopolítica, los estudios y la enseñanza, tanto de la lengua española como de las demás que componen la riqueza lingüística de nuestro país y las lenguas extranjeras con las que se busca integración cultural con otros países. De ese modo, se podría aportar al desarrollo equitativo de las lenguas que forman parte de la realidad social cotidiana colombiana. Eso sí, se requiere dedicación investigativa interdisciplinaria para la construcción de una política lingüística-cultural funcional e incluyente.

Las propuestas sistemáticas de Mahecha (2015), Tovar (1999, citadas en Mahecha 2015) y Rico (1998) trazan un camino que permite la formulación de una política lingüística acorde con la realidad sociolingüística y cultural colombiana. Debido a las dinámicas neoliberales, a los procesos de acreditación institucionales, el trabajo glotopolítico se ha limitado a dos frentes: el trabajo con el inglés LE (p. ej., Colombia Very Well) y el trabajo con el español LE (una rama importante de trabajo en el Instituto 
Caro y Cuervo). Alrededor de dichos frentes surgen, de manera discreta, los trabajos glotopolíticos con las demás lenguas nacionales.

Merecen un comentario aparte tres acciones glotopolíticas que se desarrollan actualmente en la Universidad Surcolombiana (Neiva): (i) la reglamentación oficial para determinar la competencia en español como segunda lengua, como requisito de idioma extranjero, para estudiantes de pueblos indígenas y sordos de programas de pregrado de la Universidad Surcolombiana (Acuerdo del Consejo Académico, número 027 del 14 de mayo de 2019), (ii) el desarrollo de una propuesta didáctica para la aplicación de la prueba de español a los estudiantes de pueblos indígenas y sordos de programas de pregrado de la Universidad Surcolombiana (trabajo de grado en curso) y (iii) el diseño e implementación curricular de un curso electivo de Glotopolítica para la Licenciatura en Ciencias Sociales.

\section{Referencias}

Alonso, J., Martin, J. y Gallo, B. (2015). El nivel de inglés después de cursar educación superior en Colombia: una comparación de distribuciones. Revista de Economía Institucional. 17,33 .

Areiza, R., Cisneros-Estupiñán, M. y Tabares, L. (2006). Hacia una nueva visión sociolingǘstica. Bogotá: ECOE.

Areiza, R., Cisneros-Estupiñán, M. y Tabares, L. (2012). Sociolingüística: enfoques pragmático y variacionista (1.a ed.). Bogotá: ECOE.

Areiza, R., Cisneros-Estupiñán, M. y Tabares, L. (2018). Sociolingüística: enfoques pragmático y variacionista (2.a ed.) Bogotá: ECOE.

Arismendi, F. (2016). La competencia plurilingüe y pluricultural en la formación de futuros docentes en lenguas extranjeras en una universidad pública en Colombia. Folios, 44, 109-125.

Arnoux, E. N. de (2000). La glotopolítica: transformaciones de un campo disciplinario. En Lenguajes: teorías y práctica (pp. 3-27). Buenos Aires: Secretaría de Educación, GCBA.

Arnoux, E. N. de (2008). Los discursos sobre la nación y el lenguaje en la formación del Estado (Chile, 1842-1862). Estudio glotopolítico. Buenos Aires: Santiago Arcos.

Arnoux, E. N. de (2016). La perspectiva glotopolítica en el estudio de los instrumentos lingüísticos: aspectos teóricos y metodológicos. Matraga, 32(38), 18-42. 
Arnoux, E. N. y Bein, R. (Eds.) (2015). Política lingüística y enseñanza de lenguas. Buenos Aires: Biblos.

Arnoux, E. y Del Valle, J. (2010). Las representaciones ideológicas del lenguaje. Discurso glotopolítico y panhispanismo. Spanish in Context, 7(1), 1-24.

Asociación de Lingüística y Filología de América Latina -ALFAL-. (2017). Convocatoria: Por una ciencia y una educación superior pública, gratuita, crítica, humanista e intercultural, basada en modelos plurilingües de investigación y docencia. En XVIII Congreso Internacional de ALFAL, Bogotá, D. C., Colombia, 27 de julio de 2017.

Baggioni, D. (1986). Préhistoire de la glottopolitique dans la linguistique européenne, de J. G. Herder au Cercle linguistique de Prague. Langages, 83, 35-51.

Buitrago Y., García D. y García J. (2011). Estado del arte de ELE en Colombia, una mirada holística: metodologías y enfoques, material didáctico, variedades lingüísticas y dificultades en el área. Marco ELE - Revista de Didáctica Español como Lengua Extranjera, (13). Recuperado de http://marcoele.com/descargas/13/garcia-garcia-buitrago_colombia.pdf

Calvet, L.-J. (1987). La guerre des langues et les politiques Linguistiques. Paris: Payot.

Calvet, Louis-Jean (1993). La sociolinguistique. Paris: PUF. Collection Que sais-je? [Traducción española inédita de Miguel Ángel Mahecha.]

Certeau M. de, Julia D., y Revel J. (1975) Une politique de la langue. La Révolution française et les patois. Paris: Gallimard.

Chaves-0'Flynn, Carolina (2017). Lengua y violencias en Colombia: discursos sobre patrimonio lingüístico (-2010). CUNY Academic Works. (Tesis de PhD). Recuperado de https:// academicworks.cuny.edu/gc_etds/1916

Cisneros-Estupiñán, M. (2016) El día de las lenguas en Colombia: una gran celebración mundial. En Palabras de apertura de la celebración del Día de las Lenguas. Universidad Tecnológica de Pereira. Pereira. Inédito.

Cisneros-Estupiñán, M. y Olave-Arias, G. (2018). Introducción de los estudios glotopolíticos en la formación profesional de docentes de lenguaje en Colombia. Proyecto inscrito en la Vicerrectoría de Investigaciones Innovación y Extensión de la Universidad Tecnológica de Pereira, con el código CIE 4-19-3.

Cisneros-Estupiñán, M., Rojas-García, I. y Olave-Arias, G. (2016). Didáctica de la lengua materna en Colombia. Currículos y visiones docentes. Pereira: Universidad Tecnológica de Pereira. 
Díaz C. et al. (2008). Tres historias, tres perspectivas, múltiples alternativas. Experiencias con la enseñanza del inglés en Colombia, en tiempos de globalización. Revista Educación y Pedagogía, vol. XX, n 51

Coseriu, E. (1981). La socio- y la etnolingüística: sus fundamentos y tareas. Anuario de Letras, $19,5-30$.

Del Valle, J. (2017). La perspectiva glotopolítica y la normatividad. Anuario de Glotopolítica, 1, $17-40$.

Figueroa, C. (2017). Políticas lingüísticas para estudiantes indígenas de la Universidad Nacional de Colombia, sede Bogotá. Revista Justicia y Derecho, 5, 7-49. Doi: 10.5281/zenodo.2552673

González de Pérez, M. (2011). Manual de divulgación de las lenguas indígenas de Colombia. Bogotá: Instituto Caro y Cuervo.

Guespin, L. y Marcellesi, J-B. (2019). Hacia la glotopolítica. Glottopol 32, versión española de José del Valle. Recuperado de http://glottopol.univ-rouen.fr/telecharger/numero_32/ gpl32_03guespin_marcellesi_traduccion.pdf

Hagège, Cl. (2012). Contre la pensée unique. Paris: Odile Jacob.

Instituto Caro y Cuervo (2018). Sitio web oficial. Proyectos de investigación en lingüística. Recuperado de https://www.caroycuervo.gov.co/Investigacion/

Instituto Cervantes (2010). El español, lengua global. La economía. Madrid: Grupo Santillana y Fundación telefónica. Recuperado de https://cvc.cervantes.es/lengua/espanol_economia/

Landaburu, J. (1997) Historia de la traducción de la Constitución de Colombia a siete lenguas indígenas. Amerindia, (22).

Lastra, Y. (2003). Sociolingüística para hispanoamericanos. Una introducción. México: El Colegio de México.

Lozano, M. (2012). Breves notas sobre la investigación lingüística en Colombia. Cuadernos de Lingüística Hispánica, (19), 13-22.

Mahecha, M. A. (2010). Les engagements de la sociolinguistique: le cas colombien. Paideia Surcolombiana, 1(15), 109-124. Doi: https://doi.org/10.25054/01240307.1095

Mahecha, M.A. (2015). Reflexiones propedéuticas lingüística en Colombia: El caso del bilingüismo (anteproyecto de investigación para postulación). Paideia Surcolombiana, 1(20), 79-89. Doi: https://doi.org/10.25054/01240307.1195 
Mahecha, M.A. (2017). Colombia tierra querida... Presentación Dossier Colombia. Entornos, 30(2) 135-138. Recuperado de https://journalusco.edu.co/index.php/entornos/article/ view/1628/2747

Mahecha, M. A. (en prensa). La enseñanza de la lingüística en Colombia.

Ministerio de Cultura, Colombia. (2013). Diversidad lingüística en Colombia: muchas voces, resistencia cultural y agenda de nación. En Dossier para la representación de Colombia en el Smithsonian Folklife Festival Washington, EE.UU. 26 de junio a 4 de julio de 2013 Resumen Ejecutivo. https:/www.mincultura.gov.co/areas/poblaciones/noticias/ Documents/Dossier\%20Representaci\%C3\%B3n\%20Colombia\%20Smithsonian\%20 Folklife\%20Festival.pdf

Ministerio de Educación Nacional de Colombia. (2004). Programa Nacional de Bilingüismo 2004-2019. Bogotá: MEN.

Montes, J. J. (2000). Otros estudios sobre el español de Colombia. Bogotá: Instituto Caro y Cuervo.

Montes, J.J. (1995). Dialectología general e hispanoamericana. Bogotá: Instituto Caro y Cuervo.

Oróstegui, S. (2008, jun.) Traducción de la Constitución colombiana de 1991 a siete lenguas vernáculas. Reflexión Política, 10(19), 164-175.

Pardo, F. (2001). ¿Por qué son necesarios el aprendizaje y la enseñanza del inglés en Colombia? Interlenguajes, (21), 83-96.

Pardo, N. (2011). Prólogo. Aproximación al estado del arte de los estudios del discurso. En J. Ruiz (comp.), Aproximaciones interdisciplinares al estado de los estudios del discurso (pp. 19-50). Bogotá: Universidad Nacional de Colombia.

Patiño, C. (2000). Sobre etnolingüística y otros temas. Bogotá: Instituto Caro y Cuervo.

Pineda, R. (1997). La Constitución de 1991 y la perspectiva del multiculturalismo en Colombia. En Memorias del IX Congreso de la Asociación de Colombianistas. Colombia en el contexto. Bogotá, Instituto Caro y Cuervo, Universidad de los Andes.

Pineda, R. (2000). El derecho a la lengua. Una historia de la política lingüística en Colombia. Bogotá: Universidad de los Andes.

Revista Glottopol. (2019, julio) Langage et luttes sociales dans l'espace hispano-lusophone [édition bilingue: Lenguaje y luchas sociales en el espacio hispano-lusófono]. Revista Glottopol, (32). 
Rico, A. (1981). Problèmes sociolinguistiques de la société colombienne. (Thèse de Maîtrise). Université de Grenoble, France.

Rico, A. (1994). Bilingüismo, bilingualidad y educación bilingüe. Revista Universidad del Quindio, 3(4).

Rico, A. (1998). Enseñanza y aprendizaje de lenguas extranjeras en primaria. Armenia: Oficina de Publicaciones Universidad del Quindío.

Rodríguez, Y. (1996). Los semihablantes bilingües: habilidad e interacción comunicativas. Cuadernos del Seminario Andrés Bello 8. Bogotá: Instituto Caro y Cuervo.

Soler, S. (comp.) (2014). Panorama de los estudios del discurso en Colombia. Bogotá: Universidad Distrital.

Tovar, L. (1999). Bases para una verdadera planeación lingüística en Colombia. En A.M. Truscott y L. Tovar (eds.) Perspectivas recientes del bilingüismo y de la educación bilingüe en Colombia (pp. 223-238). Cali: Universidad del Valle.

Trillos, M. (1998). Bilingüismo desigual en las escuelas de la Sierra Nevada de Santa Marta. Barranquilla: Fondo Editorial de la Universidad del Atlántico.

Trillos, M. (2001). Por una educación para la diversidad. Nómadas, (15), 162-177.

Trillos, M. y Etxebarria, M. (2002). Legislación, política lingüística y multilingüismo en Colombia. En Ponencia presentada en el Congreso Mundial sobre Políticas Lingüísticas Linguapax IX, celebrado en Barcelona, España, del 16 al 20 de abril del 2002. Recuperado de http:// www.linguapax.org/wp-content/uploads/2015/07/CMPL2002_T2_TrillosEtxebarria.pdf.

UNICEF (2009). Atlas sociolingüístico de los pueblos indígenas. Recuperado de https://www. unicef.es/prensa/unicef-presenta-el-atlas-sociolinguistico-de-pueblos-indigenas-enamerica-latina

Universidad Surcolombiana, Consejo Académico. (2019). Acuerdo número 027 del 14 de mayo. Por el cual se reglamenta la competencia en español como segunda lengua, como requisito de idioma extranjero, para estudiantes de pueblos indígenas y sordos de programas de pregrado de la Universidad Surcolombiana. Recuperado de https://www.usco.edu.co/ archivosUsuarios/19/publicacion/consejo_academico/acuerdo/acuerdo_027_de_2019. pdf

Vázquez, G. (2008). La lengua española, ¿Herencia cultural o proyecto político-económico?: Debates en el Congreso Literario Hispanoamericano de 1892. Revista Signos, 41(66), 81-106.

Villa, V. (2002). Política idiomática en Colombia: visión sociolingüística. Medellín: Editorial Universidad de Antioquia. 